\title{
Human Immunodeficiency Virus Infection and the Endocrine System
}

\author{
Dana Zaid ${ }^{1}$, Yona Greenman ${ }^{1,2}$ \\ ${ }^{1}$ Institute of Endocrinology, Metabolism and Hypertension, Tel Aviv-Sourasky Medical Center; ${ }^{2}$ Sackler Faculty of Medicine, \\ Tel Aviv University, Tel Aviv, Israel
}

In the current era of effective antiretroviral therapies (ARTs), human immunodeficiency virus (HIV) infection became a chronic disorder that requires long term follow-up. Among other medical issues, these patients may develop endocrine problems, specific to HIV infection and its treatment. The purpose of this review is to give an overview of common endocrine complications associated with HIV infection, and to propose diagnostic and therapeutic strategies. HIV can affect the endocrine system at several levels. Adrenal and gonadal dysfunction, osteoporosis with increased fracture risk, dyslipidemia with increased cardiovascular risk, are some of the endocrine disorders prevalent in HIV-infected patients that may negatively influence quality of life, and increase morbidity and mortality. While ARTs have dramatically increased life expectancy in the HIV-infected population, they are not devoid of adverse effects, including endocrine dysfunction. Physicians caring for HIV-infected patients should be knowledgeable and exercise a high index of suspicion for the diagnosis of endocrine abnormalities, and in particular be aware of those that can be life threatening. Endocrine evaluation should follow the same strategies as in the general population, including prevention, early detection, and treatment.

Keywords: HIV; Anti-retroviral agents; Endocrine system diseases; HIV-associated lipodystrophy syndrome; Diabetes mellitus; Hyperlipidemias; Osteoporosis

\section{INTRODUCTION}

Human immunodeficiency virus (HIV) affects up to $0.8 \%$ of the adult population worldwide. Although there has been a significant decrease in the number of new infections since the peak of the epidemic in 1996, in many parts of the world, especially in sub-Saharan Africa, HIV is still highly prevalent, with an estimated 23.5 million people infected [1]. The number of HIV-infected patients is rising in Asia and other parts of the world.

Endocrine dysfunction is well recognized in HIV-infected patients. Pituitary, adrenal, gonadal, thyroid, bone, and metabolic disorders have all been reported. HIV itself, related opportunis-

Received: 3 March 2019, Revised: 24 April 2019, Accepted: 13 May 2019

Corresponding author: Yona Greenman

Institute of Endocrinology, Metabolism and Hypertension, Tel Aviv-Sourasky Medical Center, 6 Weizmann St, Tel Aviv 64239, Israel

Tel: +972-3-6973899, Fax: +972-3-6973503, E-mail: Yonagr@tlvmc.gov.il tic infections, cytokines, immune activation, and antiretroviral therapy (ART) all may have adverse effects on endocrine function.

At the onset of the acquired immune deficiency (AIDS) epidemic, endocrine dysfunction was mainly a result of opportunistic infections, neoplasms, including HIV-related malignancies, and concomitant systemic illness. With the development of ART and its widespread use, there was a decline in the incidence of opportunistic infection- and neoplasm-related endocrinopathies. Chronic metabolic complications of HIV therapy, including insulin resistance and diabetes mellitus (DM), dyslipidemia, alterations in body fat distribution, hypogonadism and

Copyright $\odot 2019$ Korean Endocrine Society

This is an Open Access article distributed under the terms of the Creative Commons Attribution Non-Commercial License (http://creativecommons.org/ licenses/by-nc/4.0/) which permits unrestricted non-commercial use, distribution, and reproduction in any medium, provided the original work is properly cited. 
bone disease, and their management, became increasingly important, considering the prolonged life expectancy associated with ART.

In this review we address common endocrine issues relevant to the HIV-infected population in the ART era. Cases in which diagnosis and management of HIV-infected patients differ from those for non-HIV-infected patients are emphasized.

\section{PITUITARY FUNCTION}

Opportunistic infections and neoplasms involving the pituitary gland, mainly lymphoma, are uncommon in the ART era. Anterior pituitary function is preserved in most cases. Dynamic testing of hypothalamo-pituitary-target organ axed are usually normal in HIV-infected patients [2]. Post stimulation peak levels of growth hormone $(\mathrm{GH})$, prolactin, thyroid stimulating hormone, and adrenocorticotropic hormone (ACTH) may be even mildly increased, but the mechanism for this altered response remains unknow [3].

Despite the normal gonadotropin response to gonadotropin releasing hormone $(\mathrm{GnRH})$ stimulation, hypogonadotrophic hypogonadism is common in HIV-infected patients [4] indicating hypothalamic dysfunction. Multifactorial factors, including chronic systemic illness, may lead to suppression of the hypothalamic-pituitary-gonadal axis (HPG).

Hyperprolactinemia has been reported [5]. The cause of high prolactin levels, when documented, is basically unknown. Immunologic dysregulation, as well as the presence of macroprolactin have been suggested as possible mechanisms [6].

GH secretion is not affected by HIV infection but is altered according to the individual's nutritional status and changes in body composition. Similarly to other states of undernutrition, AIDS wasting is associated with a GH-resistant state, characterized by decreased levels of insulin-like growth factor 1 (IGF-1) despite high GH levels [7].

Patients with HIV-associated lipodystrophy, characterized by increased visceral adiposity and decreased subcutaneous fat, were found to have characteristic abnormalities: GH pulse frequency and IGF-1 levels remain normal, but basal and mean overnight GH concentrations, as well as GH pulse amplitude are lower than in patients with HIV without lipodystrophy. These alterations possibly occur as a result of increased somatostatin tone or decreased growth hormone releasing hormone (GHRH) secretion [8]. Decreased ghrelin, increased free fatty acids and insulin resistance may also play a role [8]. Reduced $\mathrm{GH}$ levels are inversely correlated with visceral obesity in HIV lipodystrophy, as also seen in non-HIV-infected population [8]. Based on GHRH and arginine stimulation test, up to one third of HIV-infected patients show evidence of biochemical growth hormone deficiency (GHD), albeit to a lesser degree compared with patients with structural pituitary disease [9]. Biochemical GHD is more common in HIV-infected men than women, which may be due to differences in adipose tissue distribution pattern. Diagnosis of GHD by stimulatory testing does not necessarily indicates clinical GHD. It is not clear at this point how GHD in HIV patients should be managed, or in which circumstance GH replacement is indicated. The use of recombinant $\mathrm{GH}$ in the context of treatment of lipodystrophy will be discussed in a following section.

Hypothalamic pituitary adrenal (HPA) axis and thyroid axis alterations will be discussed in subsequent adrenal function and thyroid function sections respectively. Primary disorders affecting posterior pituitary function are rare. Syndrome of inappropriate antidiuretic hormone secretion is common, usually secondary to infections, tumors, or medications.

\section{Effect of medications on pituitary function}

Significant elevations in prolactin levels, accompanied by galactorrhea, were reported in four HIV-infected patients treated with protease inhibitors (PIs), although three of them had received other medications known to cause hyperprolactinemia. The mechanism of PIs effect on prolactin is unclear and may relate to direct stimulation of prolactin secretion by specific PIs or effects on the P450 system to potentiate the dopamine antagonistic effect of other drugs [10]. The use of opiates may be associated with hyperprolactinemia as well as suppression of the HPA and HPG axes.

\section{ADRENAL FUNCTION}

\section{Adrenal insufficiency}

Adrenal insufficiency was one of the first endocrinopathies reported in HIV-infected patients. Although clinically significant adrenal dysfunction is uncommon in AIDS patients, mild impairments in adrenal reserve may be seen.

Biochemical evidence of adrenal insufficiency is common in hospitalized AIDS patients. In an early study, $17 \%$ of 74 patients had inadequate adrenal response when screened with a cosyntropin stimulation test. In contrast, only $4 \%$ of AIDS patients, experience clinical symptoms of hypoadrenalism [11].

Opportunistic infections are the main cause for impaired adrenal function in patients with advanced HIV disease, among 
which, cytomegalovirus (CMV) is the most common pathogen. CMV adrenalitis is seen in approximately $40 \%$ to $90 \%$ of CMV infected AIDS patients at autopsy. However, adrenocortical tissue destruction by CMV is usually less than $50 \%$ and therefore unlikely to cause adrenal insufficiency. Nowadays, CMV disease is rare in patients on potent ARTs [12].

Other infectious organisms causing adrenal insufficiency in the context of HIV are Mycobacterium tuberculosis, Mycobacterium avium intracellulare, and Cryptococcus. In addition, hemorrhage, idiopathic inflammation, or tissue destruction can cause adrenal insufficiency [11]. Secondary adrenal insufficiency may be caused in rare instances by opportunistic infection (e.g., toxoplasmosis, Cryptococcus, and CMV) involving the pituitary gland.

Clinical diagnosis of adrenal insufficiency is complex, as nonspecific complains such as fatigue and weight loss are common. Although adrenal insufficiency is uncommon, appropriate testing should be performed using a low threshold of clinical suspicion, in view of the severe mortality rate associated with this condition. Treatment with glucocorticoid replacement, coupled with mineralocorticoid supplementation in cases of primary adrenal insufficiency, should follow the same principles used to treat other causes of adrenal failure.

On the other hand, high serum cortisol levels may be seen in HIV-infected patients, most commonly in the context of a stress response, low weight, or advanced degree of illness. Some studies demonstrated a reduced dehydroepiandrosterone/cortisol ratio on cosyntropin testing, suggesting a possible intra-adrenal shunting toward cortisol synthesis, potentially as a result of 17,20-lyase dysfunction [13].

Non-pituitary factors such as cytokines, may promote adrenal steroidogenesis leading to increased cortisol levels [14]. In cases of advanced HIV infection, symptoms of adrenal insufficiency may be present despite high cortisol and ACTH levels, suggesting a glucocorticoid resistance state [2]. Finally, in the early AIDS epidemic hyporeninemic hypoaldosteronism was reported in a small case series [15].

\section{Effect of medications on adrenal function}

Anti-fungal, appetite stimulators and anti-tuberculous drugs are often used in AIDS or HIV-infected patients. Several of these drugs may affect adrenal function directly or through interaction with ART.

The antifungal drug ketoconazole inhibits side-chain cleavage enzyme and 11- $\beta$-hydroxylase in the steroidogenesis cascade, thus inhibiting cortisol synthesis and potentially leading to adre- nal insufficiency when used in high dosages. Such effects are generally not seen with fluconazole, itraconazole and more recently introduced imidazole derivatives, which are more commonly used today as antifungal agents [16].

Phenytoin and rifampin are strong cytochrome P450 enzyme 3A4 (CYP3A4) inducers and may affect cortisol metabolism. In patients with reduced adrenal reserve, adrenal insufficiency may be precipitated by rifampin given for treatment of tuberculosis [16].

Megestrol acetate, used as an appetite stimulant, is a potent synthetic progestational derivative with glucocorticoid properties. Due to its feedback inhibition of pituitary ACTH secretion, sudden withdrawal of megestrol acetate may precipitate adrenal insufficiency. It is essential to evaluate the integrity of the HPA axis before treatment interruption, to allow for physiologic glucocorticoid replacement as needed [16].

Perhaps the current most common cause for adrenal dysfunction is the interaction between ART and glucocorticoids [17]. Ritonavir or cobicistat are potent inhibitors of CYP3A4 and are often given in conjunction with some HIV medications (specifically certain PIs: atazanavir, darunavir, lopinavir) and integrase inhibitors (elvitegravir) to boost concentrations of these drugs and allow once-daily dosing. Many glucocorticoids are metabolized by CYP3A4 pathway and, as a result, levels of glucocorticoids can increase when co-administered with potent CYP3A4 inhibitors [17]. One common example is the development of iatrogenic Cushing syndrome in ritonavir treated patients that concomitantly receive inhaled fluticasone as a treatment for asthma. Inhibition of CYP3A4 by ritonavir leads to increased levels of fluticasone that is metabolized by this cytochrome enzyme. Therefore, the use of steroids that are not metabolized by CYP3A4 such as beclomethasone is recommended in asthmatic patients who are treated with ritonavir or cobicistat [17]. In patients presenting with symptoms of adrenal excess and evidence of low cortisol and ACTH levels, interactions between exogenous steroids and ART should be suspected.

Physicians should be aware that other routes of glucocorticoid administration may also lead to secondary adrenal insufficiency. It has been reported that approximately $5 \%$ of patients receiving intra-articular steroids while on PIs, particularly ritonavir, developed sustained HPA axis suppression. Intra-articular triamcinolone may suppress the HPA axis even after a single injection in such patients, with increased risk when multiple doses are administered during a period of several months [18]. Adrenal axis recovery after a single injection of triamcinolone can take many months, with slow improvement of the metabolic 
profile.

If there is persistent suppression on endogenous cortisol secretion after the cessation of exogenous glucocorticoids, adequate replacement therapy is mandatory. Inhibition of CYP3A4 in the setting of ritonavir co-administration, will also affect prednisolone metabolism such that levels are increased by approximately $30 \%$. Hence, lower prednisolone doses should be prescribed (e.g., 2 to $4 \mathrm{mg} /$ day) to avoid over-replacement. If possible, from an HIV-management standpoint, consideration should be given to interrupt treatment with the CYP3A4 inhibitor.

\section{GONADAL FUNCTION}

\section{Male hypogonadism}

Hypogonadism has been recognized among HIV-infected men early on in the HIV epidemic. Despite increased efficacy of HIV treatment, the prevalence of hypogonadism, mainly secondary hypogonadism, is higher in HIV-infected men compared with non-infected controls, in the order of $9 \%$ to $16 \%$ according to recent studies [19].

The leading cause of gonadal dysfunction in HIV-infected men relates to the effects of severe illness, weight loss, and undernutrition on gonadotropin secretion. Predisposing factors include age, obesity, and insulin resistance, particularly in men with visceral adiposity.

In HIV-infected patients with advanced disease secondary hypogonadism may be caused by opportunistic infections affecting the pituitary or hypothalamus. In such patients a pituitary/hypothalamic magnetic resonance imaging is recommended [20].

In HIV-infected men, as in the general population, chronic $\mathrm{HCV}$ infection resulting in chronic liver disease is associated with hypogonadism [21]. In this setting, the diagnosis of hypogonadism may be confounded by the characteristically elevated sex hormone binding globulin (SHBG) levels found in patients with chronic liver disorders.

Primary hypogonadism is reported less often. In a large Italian cohort, Rochira et al. [19] reported low morning testosterone associated with elevated gonadotropin levels in $16 \%$ of young patients, with a median age of 45 years. Possible mechanisms causing primary hypogonadism may involve direct cytokine effects on the testes. Tumor necrosis factor (TNF) inhibition of side-chain cleavage enzyme, as well as interleukin 1 (IL-1) inhibition of Leydig cell steroidogenesis and luteinizing hormone binding to the Leydig cell have been reported [19].

Although up to $25 \%$ of AIDS patients suffer opportunistic in- fections, including CMV and toxoplasmosis, involvement of the testes in these cases have rarely been reported. Data suggesting development of primary hypogonadism secondary to testes involvement by systemic neoplasms, Kaposi sarcoma, and testicular lymphoma are sparse [16]. When an infectious process is suspected in HIV-infected men presenting with primary hypogonadism, a scrotal ultrasound should be performed.

The accurate diagnosis of hypogonadism in HIV-infected patients may be challenging, particularly if based only on total testosterone measurements. Elevated SHBG levels are documented in $30 \%$ to $55 \%$ of HIV-infected patients, particularly in the presence of hepatitis $\mathrm{B}$ or $\mathrm{C}$ virus coinfection. Hence, a total testosterone value in the presence of high SHBG concentrations may underestimate the prevalence of hypogonadism in this population. Approximately as much as $30 \%$ of patients with biochemical hypogonadism will be missed by measuring only total testosterone. Therefore, the use of bioavailable or free testosterone is recommended for diagnosis [20]. If the total testosterone is in the lower half of the normal range (below $500 \mathrm{ng} / \mathrm{dL}$ in most assays) in an HIV-infected man with symptomatic hypogonadism, a free or bioavailable testosterone assay should be performed.

In addition to low testosterone levels, symptoms of androgen deficiency such as erectile dysfunction, decreased libido, fatigue, and muscle wasting should be estimated. Treatment of male hypogonadism in male HIV-infected patients follows the same principles used for the general populations. A special consideration should be given to HIV-infected men presenting with weight loss and muscle weakness associated with low testosterone levels. Short term testosterone treatment has been shown to improve muscle strength and increase body weight and lean body mass in these patients [20].

\section{Female hypogonadism}

In HIV-infected women, hypogonadism presenting as amenorrhea is common, occurring in approximately $25 \%$ of patients. Anovulation is seen in up to $50 \%$ of HIV-infected women with reduced cluster of differentiation 4 (CD4) counts. Reduced in gonadotropin synthesis and secretion in the context of the stress of illness is the most probable culprit. Early menopause has been reported in up to $8 \%$ of HIV-infected women [22].

Reduced androgen levels are often seen in HIV-infected women. The cause of androgen deficiency in the context of HIV may be explained in part by intra-adrenal shunting toward cortisol production and away from androgen production, especially in the presence of weight loss. 


\section{Effect of medications on gonadal function}

Ketoconazole use for the treatment of fungal infections prevalent in the immune-suppressed state associated with HIV may cause hypogonadism. Inhibition of side-chain cleavage enzyme and other enzymes involved in steroidogenesis leads to a decrease in testosterone synthesis and sexual dysfunction in males. Disrupted steroidogenesis in the ovary lead to menstrual disturbances in female patients.

Gonadal function may also be affected by megestrol acetate, a synthetic progestational agent mostly used as an appetite stimulant. Megestrol acetate causes hypogonadism through suppression of gonadotropin secretion. Similarly, opiates may lead to hypogonadotrophic hypogonadism by suppressing $\mathrm{GnRH}$ secretion.

There is no consensus regarding ART effects on the hypothalamic pituitary gonadal axis. Some studies have shown that testosterone levels are restored to normal upon initiation of ART, but others showed no effect $[23,24]$. ART, particularly PIs, has been associated with male sexual dysfunction, but most patients on ART have normal testosterone levels.

\section{THYROID FUNCTION}

Alterations in thyroid function tests are commonly seen in HIVinfected patients. Thyroxin binding globulin (TBG) levels are elevated in HIV-infected patients and show inverse correlation with CD4 counts [25]. Abnormal thyroid function tests may be seen in advanced disease, as found in other patients with "euthyroid sick syndrome." However, in contrary to nonthyroidal illness, reverse T3 (rT3) levels do not rise in association with decreasing T3 levels [26]. Thyroid function alterations occurring in progressive HIV disease include decreased T3 levels, increased TBG and decreased rT3 levels with increasing illness. T3 levels are often higher and rT3 levels lower in AIDS patients than expected for nonthyroidal illness. Asymptomatic HIV-infected patients with stable body weight generally maintain normal thyroid function.

As demonstrated in large screening studies, overt thyroid disease in not increased in comparison with the general population. In one study of 1,565 HIV-infected patients, the prevalence of nonthyroidal illness was $17 \%$, overt hypothyroidism $2.5 \%$, subclinical hypothyroidism $4 \%$, and overt hyperthyroidism $1 \%$ [27]. Thyroid dysfunction has been described in the context of the immune reconstitution syndrome (IRIS). In this case, improved immune function secondary to ART treatment, leads to production of new immune cells targeting thyroid antigens, typ- ically 12 to 36 months after ART is initiated [28]. Graves' disease is most often reported in this context. The estimated prevalence of immune reconstitution associated thyroid disease was reported to be $3 \%$ for women and $0.2 \%$ for men [29].

Opportunistic infections in HIV-infected patients may lead to thyroid dysfunction Pneumocystis thyroiditis has been reported to cause a painful thyroiditis-like picture. CMV, M. avium intracellulare, Cryptococcus, and Kaposi sarcoma have been isolated in thyroid tissue obtained by autopsy, but have not been related to clinical symptoms. Thyroidal abscesses from Aspergillus and Rhodococcus equi leading to thyroid dysfunction have also been reported. Opportunistic infections with toxoplasma gondii and CMV involving the hypothalamus and pituitary may lead to secondary hypothyroidism.

\section{Effect of medications on thyroidal function}

Subclinical hypothyroidism, is more frequent in patients on ART compared with ART-naïve patients [30]. Rifampin and ritonavir enhance hepatic clearance of thyroxine and can precipitate hypothyroidism in patients with marginal thyroid reserve. Patients receiving thyroid replacement therapy may require higher doses in this setting. Interferon treatment is associated with an increased incidence of hypothyroidism, while Graves' disease has been described in association with IL-2 therapy in HIV-infected patients.

\section{BONE DISORDERS}

Multiple studies have shown a higher prevalence of osteoporosis and increased risk of fragility fractures in HIV-infected patients compared with healthy subjects. Current data suggest that immunologic factors such as activation of T-cells, low CD4 cell count, and coinfection with hepatitis $\mathrm{B}$ and $\mathrm{C}$ are strongly associated with reduced bone density, particularly in women.

HIV infection in itself is considered a risk factor for osteoporosis and fragility fractures. It causes T-cell activation and production of pro-inflammatory cytokines including TNF- $\alpha$, IL-6, receptor activator of nuclear factor kappa-B ligand (RANKL), and other soluble immune factors that enhance activity of osteoclasts, resulting in increased bone resorption [31]. The levels of endogenous inhibitors of osteoclastogenesis including osteoprotegrin and interferon $\mathrm{\gamma}$ are downregulated in advanced HIV infection. HIV proteins such as Tag and Nef reduce the amount of mesenchymal stem cell (MSC) precursors that can proliferate into osteoblasts by inducing MSC senescence, leading to decreased bone formation [16]. 
Endocrine factors including hypogonadism, relative GH deficiency and vitamin D deficiency may further contribute to reduced bone density in HIV-infected patients. Lipoatrophy may mediate bone loss as well, through a complex relationship between central signaling of adipocyte hormones and bone [16]. Impaired parathyroid hormone (PTH) secretion and action have also been reported in HIV-infected patients [32].

In view of the above, dual-energy X-ray absorptiometry screening is recommended earlier (postmenopausal women and men $>50$ years of age) in HIV-infected patients relative to the general population ( $>65$ for men and $>70$ years of age for women) [33].

\section{Effect of medications on bone health}

The initiation of ART is associated with a decrease in bone mineral density (BMD) of $2 \%$ to $6 \%$ over a time period of 96 weeks. This bone loss is not reversible and is independent of the specific ART regimen used [33].

Some ART medications, particularly tenofovir disoproxil fumarate (TDF), and PIs have direct deleterious effects on BMD. TDF may augment phosphate reabsorption in the proximal tubule (Fanconi's syndrome), leading to secondary increase in PTH and bone turnover. Osteomalacia is an additional skeletal complication that occurs in this context [34]. A different formulation of tenofovir, tenofovir alafenamide (TAF), is less deleterious to bone compared with TDF. Vitamin D deficiency may occur through various mechanisms: impairment of $1-\alpha$-hydroxylase may be secondary to treatment with PIs, whereas efavirenz may reduce cytochrome P450 2RI expression, leading to decreased 25-hydroxylation of vitamin D. Further, conversion of vitamin $\mathrm{D}$ into its inactive metabolites is promoted by the same mechanism [35].

IRIS following the initiation of ART has also been implicated in bone loss. The rapid improvement in immune function leads to an increase in cytokine levels, as well as to systemic or local inflammation that may also contribute to bone loss. The magnitude of CD4-recovery has been shown to positively correlate with the increase in bone resorption markers [16].

\section{Treatment of osteoporosis in HIV-infected patients}

Strategies to attenuate bone loss include calcium and vitamin D supplementation, as well as life style changes such as smoking cessation and weight-bearing exercise. Co-morbidities known to adversely affect bone health such as hypogonadism should be corrected. Importantly, substitution of TDF and/or PIs by other treatment regimens is an important strategy leading to improved bone health in HIV-infected patients [36]. Switching TDF to other ART regimens (including TAF, abacavir, or raltegravir) has been shown to increase BMD by $1 \%$ to $3 \%$ over 48 to 96 weeks, but effects on fracture risk are not available.

Bisphosphonates are the first line therapy for osteoporosis in HIV-infected patients. Alendronate and zoledronate, have been evaluated for the treatment of osteoporosis in HIV patients. Inhibition of bone turnover markers and an increase in bone density in lumbar spine and hip were consistently achieved, but there is no data on fracture prevention. A single dose of intravenous zoledronic acid prior to initiation of ART has been shown to prevent treatment-associated bone loss [36]. Studies to evaluate other, non-bisphosphonate medications such as PTH analogs and denosumab were not specifically studied in HIV-infected patients [16].

\section{METABOLIC CHANGES}

\section{Dyslipidemia}

The prevalence of cardiovascular disease (CVD) in HIV-infected patients is higher compared with HIV-uninfected controls. This can be attributable to an increased prevalence of traditional CVD risk factors in addition to the effects of chronic inflammation. Dyslipidemia has been documented in up to $54 \%$ of patients with HIV [16].

Lipid abnormalities are particularly common in patients with lipodystrophy syndrome, characterized by changes in fat distribution, with increased visceral and upper trunk fat. Hypertriglyceridemia, related in part to an increased secretion and decreased clearance of very low-density lipoprotein (VLDL), has long been associated with HIV infection and was observed prior to the introduction of ART [37,38]. As reported in longitudinal studies, a significant decrease in total, high-density lipoprotein (HDL), and low-density lipoprotein (LDL) cholesterol are observed following seroconversion [39]. With ART initiation, total and LDL cholesterol return to baseline, pre-infection levels, but low HDL levels usually persist [39]. Among patients with changes in fat distribution, 57\% had hypertriglyceridemia and $46 \%$ low HDL in comparison with a matched cohort from the Framingham Offspring Study [40]. Some studies suggest an increase in atherogenic small dense LDL in patients with HIV associated lipodystrophy.

\section{Effect of medications on lipid profile}

ART play a role in the development of dyslipidemia, although modern regimens have less metabolic toxicities than those used 
in the early ART era. Treatment with PIs may be associated with dyslipidemia in $28 \%$ to $80 \%$ of patients. Dyslipidemia is more common with lopinavir/ritonavir, tipranavir and fosamprenavir, but less frequently associated with darunavir and atazanavir use. Possible mechanisms of PI induced dyslipidemia include inhibition of adipocyte differentiation and lipogenesis, decreased clearance of chylomicrons and VLDL, and increased synthesis of triglycerides by the liver. Among the non-nucleoside reverse transcriptase inhibitors (NNRTIs) the most common drug associated with dyslipidemia is efavirenz, in contrast with rilpivirine, etravirine, and nevirapine that in general do not cause this side effect.

Thymidine analog nucleoside reverse transcriptase inhibitors (NRTIs; stavudine, didanosine, and zidovudine) which are rarely used in the current ART regimens, are associated with lipid dyscrasias and lipoatrophy, while newer NRTIs, abacavir and tenofovir, have neutral or even favorable effects on lipids profile. For example, the combination of TDF and emtricitabine may lower total and LDL cholesterol and triglycerides. Integrase standard transfer inhibitors (INSTIs) such as raltegravir and dolutegravir are more lipid neutral [16].

\section{Treatment of dyslipidemia in HIV-infected patients}

Statins are the first-line therapy, as in case of dyslipidemia in non-HIV-infected individuals. It is unknown whether the threshold for the use of statins should be lower in HIV-infected patients, considering the higher risk of CVD events and a higher burden of subclinical atherosclerosis compared with matched HIV-uninfected persons. CVD risk calculators used for the general population likely underestimate this risk in HIV-infected patients; therefore, a risk-calculation tool specific to the HIV-infected population has been developed (http://www.chip.dk/ TOOLS).

It is important to be aware of interactions between certain statins and ARTs. Co-administration of potent CYP3A4 inhibitors, such as ritonavir or cobicistat with statins that are extensively metabolized by this cytochrome such as simvastatin and lovastatin is contraindicated given the risk of rhabdomiolysis. Although potent statins such as atorvastatin and rosuvastatin, are metabolized by CYP3A4 in a lesser degree, it is recommended to exercise caution and reduce their dose to avoid reaching high serum levels that may increase the risk for side effects. Pravastatin has few interactions with ARTs, with one important exception of the PI darunavir, that may increase pravastatin levels to as much as $80 \%$. Pitavastatin has no known interactions with ARTs. Ezetimib, fenofibrate, niacin, and omega
3 fatty acids have been studied in HIV-infected patients and in general their use have been found to be safe [41].

\section{Diabetes mellitus}

Insulin resistance and DM are not uncommon among HIV-infected patients. The reported prevalence of DM is between $2 \%$ to $14 \%$ [16]. Insulin resistance is considered the primary mechanism for impaired glucose tolerance and DM in these patients. Increased body mass index, lipodystrophy, low CD4 counts and exposure to older ARTs, including stavudine and indinavir, are predictive of DM in this patient population [42].

There are multiple mechanisms involved in the development of insulin resistance in HIV-infected patients. Abnormal fat distribution, loss of peripheral subcutaneous fat, altered cytokines (e.g., low adiponectin and leptin, increased soluble TNF receptor 1), mitochondrial dysfunction, increased lipolysis and hepatic and muscle fat accumulation may be involved [43]. In addition, altered function of CD4+ and CD8+ T-cells may impair glycolysis. Damage to the structural barrier of the gastrointestinal tract may occur in chronic HIV infection, leading to increased microbial translocation. The associated inflammation may persist even after ART initiation and despite viral suppression. The chronic inflammation state, is associated with metabolic dysfunction, increased risk of development DM and cardiovascular morbidity [44].

Hemoglobin A1c (HbAlc) underestimates glycemia in HIVinfected patients by $0.2 \%$ to $0.5 \%$. This is thought to be due to low-grade hemolysis, higher mean corpuscular volume, NRTI use (specifically abacavir), and reduced CD4 count. It is suggested to obtain HbA1c prior to and within 1 to 3 months after starting ART and repeat testing every 6 to 12 months while using $\mathrm{HbA} 1 \mathrm{c}$ threshold cutoff of $5.8 \%$ for the diagnosis of DM [16].

\section{Effect of medications on glucose balance}

Before the availability of ART, most cases of DM occurred in patients with Pneumocystis jirovecii infection treated with pentamidine. Pentamidine is known to destroy pancreatic $\beta$-cells, leading to insulin deficiency and ketoacidosis-prone diabetes.

PIs increase insulin resistance by inhibiting the transport function of glucose transporter type 4 thus decreasing glucose uptake and insulin secretion. Newer PIs (darunavir and atazanavir) have limited influence on insulin sensitivity [16]. Some NRTIs are associated with insulin resistance, due to mitochondrial toxicity or through effects on subcutaneous fat [45].

If the patient develops DM while treated with ARTs, consid- 
eration should be given to switch the therapeutic regimen, particularly if it consists of lopinavir/ritonavir or a thymidine ana$\log$ (zidovudine, stavudine).

Type 2 diabetes should be managed in the HIV-infected population according to current guidelines for the general population. There are few interactions between DM medications and PIs, with the exception of saxagliptin, which should be avoided. On the other hand, dolutegravir may increase metformin concentration, and in case of co-administration the total daily dose of metformin should be limited to $1,000 \mathrm{mg}$ and careful monitoring is required [16].

\section{Lipodystrophy}

Prior to the availability of effective ART, severe wasting and decreased levels of cholesterol were common metabolic abnormalities in advanced AIDS patients [46]. After the introduction of effective ART, the prevailing metabolic abnormalities are secondary to central fat accumulation and peripheral fat loss. The term "HIV-associated lipodystrophy syndrome" was coined, but today it is clear that this is not a single syndrome but rather the co-occurrence of phenotypes that vary from person to person. Some individuals present with lipoatrophy, others have fat accumulation, and some have a mixed picture [47]. These morphologic changes are associated with metabolic complications such as disorders in glucose and lipid metabolism.

\section{Lipoatrophy}

Lipoatrophy involves the loss of subcutaneous fat in the face, arms, legs, abdomen, and/or buttocks. Unlike AIDS-associated wasting, loss of subcutaneous fat in lipoatrophy is not associated with loss of lean body mass. Metabolic complications including dyslipidemia and dysglycemia are common in patients with lipoatrophy, and its presence should prompt evaluation for these conditions. HIV disease severity and host factors (age, baseline body type) may influence the risk of lipoatrophy, but the main etiology is iatrogenic.

\section{Effect of medications on lipoatrophy development}

The main risk factor for development of lipoatrophy is exposure to thymidine analogs NRTIs (stavudine more than zidovudine). Although these are not first-line NRTI drugs used today, some patients who started treatment many years ago may still be taking this medication. These medications are also still used in some resource-limited settings.

The underlying mechanism of lipoatrophy development may be a NRTI-induced inhibition of mitochondrial DNA poly- merase and mitochondrial toxicity, leading to adipocyte apoptosis. Biopsies of subcutaneous adipose tissue from patients with lipoatrophy are characterized by depletion of mitochondrial DNA, inflammation, and apoptosis [48].

Many potential drug therapies for lipoatrophy have been investigated, including uridine, thiazolidinediones, and statins, and found to be ineffective. Treatment of patient with lipoatrophy and reduced leptin levels with recombinant leptin has been shown to improve insulin resistance in small pilot studies.

\section{Fat accumulation-lipohypertrophy}

HIV-associated fat accumulation is mainly visceral, while subcutaneous fat tissue remains normal or may decrease. Fat accumulation in HIV is characteristically central, including abdominal visceral adipose tissue (VAT) deposition with increased abdominal girth, and fat accumulation in the dorsocervical area, trunk, and upper chest. This pattern of fat accumulation is often associated with dyslipidemia and insulin resistance. Patients of both genders may develop breast fat deposition or subcutaneous lipomas. Fat accumulation can occur to some degree with any ART regimen, being prevalent in up to $70 \%$ of patients [49].

Interestingly, lipoatrophy may coexist with fat accumulation. The association between lipodystrophy and metabolic abnormalities in HIV-infected patients may be mediated by changes in several adipokines. Adiponectin levels are low, secondary to decreased adipocyte differentiation [50,51]. Lower leptin levels were documented in patients with significant lipoatrophy compared with HIV-infected patients without lipoatrophy [52]. However, there is no absolute leptin threshold that is associated with metabolic abnormalities. Finally, a single nucleotide polymorphism in the resistin gene, which has been previously linked to DM in obese patients, was found in HIV-infected patients who developed significant body composition and metabolic changes on ART [53].

\section{Treatment of lipohypertrophy}

Tesamorelin, a GHRH analog, is a U.S. Food and Drug Administration-approved drug for reduction of excess VAT in patients with treated HIV infection. It increases pituitary GH release and reduces VAT by approximately 15\% within 6 months in HIV-infected patients. Long term efficacy and safety data with tesamorelin treatment is as yet not available [16].

\section{CONCLUSIONS}

In the current era of effective ART, life expectancy of treated 
HIV-infected patients is close to that of the general population. Nevertheless, HIV infection and its treatment have increased the risk of various medical complications, including those involving the endocrine system. If left undiagnosed and untreated, these complications may lead to increased morbidity and mortality. Compared to older ARTs, current ART regimens have significantly less adverse effects on lipid and glucose metabolism. Still, there is a higher risk of DM and cardiovascular events in the HIV-infected population. Hence it is important that not only endocrinologists and infectious disease specialists, but also primary care physicians be aware of the risk of endocrinopathies associated with HIV infection and ARTs, to promote earlier diagnosis and treatment of these conditions. Finally, awareness of possible drug interactions with concurrent HIV treatment is essential.

\section{CONFLICTS OF INTEREST}

No potential conflict of interest relevant to this article was reported.

\section{ORCID}

Yona Greenman https://orcid.org/0000-0003-4923-488X

\section{REFERENCES}

1. Avert. Global HIV and AIDS statistics [Internet]. Brighton: Avert; c2017 [cited 2019 Mar 26]. Available from: http:// www.avert.org/worldstats.htm.

2. Raffi F, Brisseau JM, Planchon B, Remi JP, Barrier JH, Grolleau JY. Endocrine function in $98 \mathrm{HIV}$-infected patients: a prospective study. AIDS 1991;5:729-33.

3. Wilson LD, Truong MP, Barber AR, Aoki TT. Anterior pitutiary and pitutiary-dependent target organ function in men infected with the human immunodeficiency virus. Metabolism 1996;45:738-46.

4. Poretsky L, Can S, Zumoff B. Testicular dysfunction in human immunodeficiency virus-infected men. Metabolism 1995;44:946-53.

5. Graef AS, Gonzalez SS, Baca VR, Ramirez ML, Daza LB, Blanco FF, et al. High serum prolactin levels in asymptomatic HIV-infected patients and in patients with acquired immunodeficiency syndrome. Clin Immunol Immunopathol 1994;72:390-3.

6. Ram S, Acharya S, Fernando JJ, Anderson NR, Gama R.
Serum prolactin in human immunodeficiency virus infection. Clin Lab 2004;50:617-20.

7. Heijligenberg R, Sauerwein HP, Brabant G, Endert E, Hommes MJ, Romijn JA. Circadian growth hormone secretion in asymptomatic human immune deficiency virus infection and acquired immunodeficiency syndrome. J Clin Endocrinol Metab 1996;81:4028-32.

8. Rietschel P, Hadigan C, Corcoran C, Stanley T, Neubauer G, Gertner J, et al. Assessment of growth hormone dynamics in human immunodeficiency virus-related lipodystrophy. J Clin Endocrinol Metab 2001;86:504-10.

9. Rochira V, Guaraldi G. Growth hormone deficiency and human immunodeficiency virus. Best Pract Res Clin Endocrinol Metab 2017;31:91-111.

10. Hutchinson J, Murphy M, Harries R, Skinner CJ. Galactorrhoea and hyperprolactinaemia associated with protease-inhibitors. Lancet 2000;356:1003-4.

11. Membreno L, Irony I, Dere W, Klein R, Biglieri EG, Cobb E. Adrenocortical function in acquired immunodeficiency syndrome. J Clin Endocrinol Metab 1987;65:482-7.

12. Glasgow BJ, Steinsapir KD, Anders K, Layfield LJ. Adrenal pathology in the acquired immune deficiency syndrome. Am J Clin Pathol 1985;84:594-7.

13. Grinspoon S, Corcoran C, Stanley T, Rabe J, Wilkie S. Mechanisms of androgen deficiency in human immunodeficiency virus-infected women with the wasting syndrome. J Clin Endocrinol Metab 2001;86:4120-6.

14. Villette JM, Bourin P, Doinel C, Mansour I, Fiet J, Boudou P, et al. Circadian variations in plasma levels of hypophyseal, adrenocortical and testicular hormones in men infected with human immunodeficiency virus. J Clin Endocrinol Metab 1990;70:572-7.

15. Kalin MF, Poretsky L, Seres DS, Zumoff B. Hyporeninemic hypoaldosteronism associated with acquired immune deficiency syndrome. Am J Med 1987;82:1035-8.

16. Mirza FS, Luthra P, Chirch L. Endocrinological aspects of HIV infection. J Endocrinol Invest 2018;41:881-99.

17. Elliot ER, Theodoraki A, Jain LR, Marshall NJ, Boffito M, Baldeweg SE, et al. Iatrogenic Cushing's syndrome due to drug interaction between glucocorticoids and the ritonavir or cobicistat containing HIV therapies. Clin Med 2016;16:4128.

18. Hyle EP, Wood BR, Backman ES, Noubary F, Hwang J, Lu $Z$, et al. High frequency of hypothalamic-pituitary-adrenal axis dysfunction after local corticosteroid injection in HIVinfected patients on protease inhibitor therapy. J Acquir Im- 
mune Defic Syndr 2013;63:602-8.

19. Rochira V, Zirilli L, Orlando G, Santi D, Brigante G, Diazzi $\mathrm{C}$, et al. Premature decline of serum total testosterone in HIV-infected men in the HAART-era. PLoS One 2011;6: e28512.

20. Bhasin S, Cunningham GR, Hayes FJ, Matsumoto AM, Snyder PJ, Swerdloff RS, et al. Testosterone therapy in men with androgen deficiency syndromes: an Endocrine Society clinical practice guideline. J Clin Endocrinol Metab 2010; 95:2536-59.

21. Bannister P, Handley T, Chapman C, Losowsky MS. Hypogonadism in chronic liver disease: impaired release of luteinising hormone. Br Med J 1986;293:1191-3.

22. Clark RA, Mulligan K, Stamenovic E, Chang B, Watts H, Andersen J, et al. Frequency of anovulation and early menopause among women enrolled in selected adult AIDS clinical trials group studies. J Infect Dis 2001;184:1325-7.

23. Dube MP, Parker RA, Mulligan K, Tebas P, Robbins GK, Roubenoff R, et al. Effects of potent antiretroviral therapy on free testosterone levels and fat-free mass in men in a prospective, randomized trial: A5005s, a substudy of AIDS Clinical Trials Group Study 384. Clin Infect Dis 2007;45:120-6.

24. Wunder DM, Bersinger NA, Fux CA, Mueller NJ, Hirschel B, Cavassini M, et al. Hypogonadism in HIV-1-infected men is common and does not resolve during antiretroviral therapy. Antivir Ther 2007;12:261-5.

25. Bourdoux PP, De Wit SA, Servais GM, Clumeck N, Bonnyns MA. Biochemical thyroid profile in patients infected with the human immunodeficiency virus. Thyroid 1991;1:147-9.

26. Grinspoon SK, Bilezikian JP. HIV disease and the endocrine system. N Engl J Med 1992;327:1360-5.

27. Madge S, Smith CJ, Lampe FC, Thomas M, Johnson MA, Youle M, et al. No association between HIV disease and its treatment and thyroid function. HIV Med 2007;8:22-7.

28. Hoffmann CJ, Brown TT. Thyroid function abnormalities in HIV-infected patients. Clin Infect Dis 2007;45:488-94.

29. Chen F, Day SL, Metcalfe RA, Sethi G, Kapembwa MS, Brook MG, et al. Characteristics of autoimmune thyroid disease occurring as a late complication of immune reconstitution in patients with advanced human immunodeficiency virus (HIV) disease. Medicine 2005;84:98-106.

30. Madeddu G, Spanu A, Chessa F, Calia GM, Lovigu C, Solinas $\mathrm{P}$, et al. Thyroid function in human immunodeficiency virus patients treated with highly active antiretroviral therapy (HAART): a longitudinal study. Clin Endocrinol 2006;64: 375-83.
31. Manolagas SC, Jilka RL. Bone marrow, cytokines, and bone remodeling. Emerging insights into the pathophysiology of osteoporosis. N Engl J Med 1995;332:305-11.

32. Hellman P, Albert J, Gidlund M, Klareskog L, Rastad J, Akerstrom $\mathrm{G}$, et al. Impaired parathyroid hormone release in human immunodeficiency virus infection. AIDS Res Hum Retroviruses 1994;10:391-4.

33. Brown TT, Hoy J, Borderi M, Guaraldi G, Renjifo B, Vescini F, et al. Recommendations for evaluation and management of bone disease in HIV. Clin Infect Dis 2015;60:124251.

34. Perrot S, Aslangul E, Szwebel T, Caillat-Vigneron N, Le Jeunne C. Bone pain due to fractures revealing osteomalacia related to tenofovir-induced proximal renal tubular dysfunction in a human immunodeficiency virus-infected patient. J Clin Rheumatol 2009;15:72-4.

35. Brown TT, McComsey GA. Association between initiation of antiretroviral therapy with efavirenz and decreases in 25-hydroxyvitamin D. Antivir Ther 2010;15:425-9.

36. Yin MT, Brown TT. HIV and bone complications: understudied populations and new management strategies. Curr HIV/AIDS Rep 2016;13:349-58.

37. Hellerstein MK, Grunfeld C, Wu K, Christiansen M, Kaempfer S, Kletke C, et al. Increased de novo hepatic lipogenesis in human immunodeficiency virus infection. J Clin Endocrinol Metab 1993;76:559-65.

38. Grunfeld C, Pang M, Doerrler W, Shigenaga JK, Jensen P, Feingold KR. Lipids, lipoproteins, triglyceride clearance, and cytokines in human immunodeficiency virus infection and the acquired immunodeficiency syndrome. J Clin Endocrinol Metab 1992;74:1045-52.

39. Riddler SA, Smit E, Cole SR, Li R, Chmiel JS, Dobs A, et al. Impact of HIV infection and HAART on serum lipids in men. JAMA 2003;289:2978-82.

40. Hadigan C, Meigs JB, Corcoran C, Rietschel P, Piecuch S, Basgoz N, et al. Metabolic abnormalities and cardiovascular disease risk factors in adults with human immunodeficiency virus infection and lipodystrophy. Clin Infect Dis 2001;32: 130-9.

41. Myerson M. Lipid management in human immunodeficiency virus. Endocrinol Metab Clin North Am 2016;45:141-69.

42. Rasmussen LD, Mathiesen ER, Kronborg G, Pedersen C, Gerstoft J, Obel N. Risk of diabetes mellitus in persons with and without HIV: a Danish nationwide population-based cohort study. PLoS One 2012;7:e44575.

43. Carper MJ, Cade WT, Cam M, Zhang S, Shalev A, Yarash- 
eski KE, et al. HIV-protease inhibitors induce expression of suppressor of cytokine signaling-1 in insulin-sensitive tissues and promote insulin resistance and type 2 diabetes mellitus. Am J Physiol Endocrinol Metab 2008;294:E558-67.

44. Pedersen KK, Pedersen M, Troseid M, Gaardbo JC, Lund TT, Thomsen C, et al. Microbial translocation in HIV infection is associated with dyslipidemia, insulin resistance, and risk of myocardial infarction. J Acquir Immune Defic Syndr 2013;64:425-33.

45. Fleischman A, Johnsen S, Systrom DM, Hrovat M, Farrar CT, Frontera W, et al. Effects of a nucleoside reverse transcriptase inhibitor, stavudine, on glucose disposal and mitochondrial function in muscle of healthy adults. Am J Physiol Endocrinol Metab 2007;292:E1666-73.

46. Grunfeld C, Kotler DP, Hamadeh R, Tierney A, Wang J, Pierson RN. Hypertriglyceridemia in the acquired immunodeficiency syndrome. Am J Med 1989;86:27-31.

47. Mulligan K, Parker RA, Komarow L, Grinspoon SK, Tebas P, Robbins GK, et al. Mixed patterns of changes in central and peripheral fat following initiation of antiretroviral therapy in a randomized trial. J Acquir Immune Defic Syndr 2006; 41:590-7.

48. Hammond E, McKinnon E, Nolan D. Human immunodeficiency virus treatment-induced adipose tissue pathology and lipoatrophy: prevalence and metabolic consequences. Clin Infect Dis 2010;51:591-9.

49. Jacobson DL, Knox T, Spiegelman D, Skinner S, Gorbach S, Wanke C. Prevalence of, evolution of, and risk factors for fat atrophy and fat deposition in a cohort of HIV-infected men and women. Clin Infect Dis 2005;40:1837-45.

50. Addy CL, Gavrila A, Tsiodras S, Brodovicz K, Karchmer AW, Mantzoros CS. Hypoadiponectinemia is associated with insulin resistance, hypertriglyceridemia, and fat redistribution in human immunodeficiency virus-infected patients treated with highly active antiretroviral therapy. J Clin Endocrinol Metab 2003;88:627-36.

51. Sweeney LL, Brennan AM, Mantzoros CS. The role of adipokines in relation to HIV lipodystrophy. AIDS 2007;21: 895-904.

52. Nagy GS, Tsiodras S, Martin LD, Avihingsanon A, Gavrila A, Hsu WC, et al. Human immunodeficiency virus type 1-related lipoatrophy and lipohypertrophy are associated with serum concentrations of leptin. Clin Infect Dis 2003; 36:795-802.

53. Ranade K, Geese WJ, Noor M, Flint O, Tebas P, Mulligan K, et al. Genetic analysis implicates resistin in HIV lipodystrophy. AIDS 2008;22:1561-8. 